\title{
Magnified Endoscopic Findings of Multiple White Flat Lesions: A New Subtype of Gastric Hyperplastic Polyps in the Stomach
}

\author{
Rino Hasegawa', Kenshi Yao ${ }^{1}$, Shoutomi Ihara ${ }^{2}$, Masaki Miyaoka', Takao Kanemitsu', Kenta Chuman', Go Ikezono', \\ Akikazu Hirano ${ }^{1}$, Toshiharu Ueki ${ }^{3}$, Hiroshi Tanabe ${ }^{4}$, Atsuko Ota ${ }^{4}$, Seiji Haraoka ${ }^{4}$ and Akinori Iwashita ${ }^{4}$ \\ ${ }^{1}$ Department of Endoscopy, Fukuoka University Chikushi Hospital, Fukuoka, ${ }^{2}$ Department of Cardiology, Iharakasuga Clinic, Fukuoka, \\ ${ }^{3}$ Department of Gastroenterology, Fukuoka University Chikushi Hospital, Fukuoka, ${ }^{4}$ Department of Pathology, Fukuoka University Chikushi \\ Hospital, Fukuoka, Japan
}

Background/Aims: While the occurrence of multiple whitish flat elevated lesions (MWFL) was first reported in 2007, no studies on MWFL have been published to date. The present retrospective observational study aimed to clarify the endoscopic findings and clinicopathological features of MWFL.

Methods: Subjects were consecutive patients who underwent upper gastrointestinal endoscopy as part of routine screening between April 2014 and March 2015. The conventional white-light, non-magnifying and magnifying narrow-band images were reviewed. Clinical features were compared between patients with and without MWFL.

Results: The conventional endoscopic findings of MWFL include multiple whitish, flat, and slightly elevated lesions of various sizes, mainly located in the gastric body and fundus. Narrow-band imaging enhanced the contrast of MWFL and background mucosa, and magnifying narrow-band imaging depicted a uniformly long, narrow, and elliptical marginal crypt epithelium with an unclear microvascular pattern. Histopathological findings revealed hyperplastic changes of the foveolar epithelium, and parietal cell protrusions and oxyntic gland dilatations were observed in the fundic glands, without any intestinal metaplasia. The rate of acid-reducing drug use was significantly higher in patients with MWFL than in those without $(100 \%$ [13/13] vs. 53.7\% [88/164], $p<0.001)$.

Conclusions: The present study indicated a relationship between the presence and endoscopic features of MWFL and history of acidreducing drug use. Clin Endosc 2018;51:558-562

Key Words: Multiple white flat lesions; Proton pump inhibitor; Stomach; Polyp; Magnified endoscopy findings

\section{INTRODUCTION}

The majority of benign epithelial non-neoplastic flat elevated gastric lesions are foveolar hyperplastic and fundic gland polyps. ${ }^{1}$ However, the occurrence of multiple whitish flat elevated lesions (MWFL) extending from the gastric body to the fundus was first reported by Kawaguchi et al. in $2007{ }^{2}$

Received: June 15, 2018 Revised: October 15, 2018

Accepted: October 16, 2018

Correspondence: Kenshi Yao

Department of Endoscopy, Fukuoka University Chikushi Hospital,

1-1-1 Zokumyoin, Chikushino, Fukuoka 818-8502, Japan

Tel: +81-92-921-1011, Fax: +81-92-929-2630, E-mail: yao@fukuoka-u.ac.jp

ORCID: https://orcid.org/0000-0003-0863-3649

(c) This is an Open Access article distributed under the terms of the Creative Commons Attribution Non-Commercial License (http://creativecommons.org/ licenses/by-nc/3.0) which permits unrestricted non-commercial use, distribution, and reproduction in any medium, provided the original work is properly cited.
In 2011, Kamada et al. described the same lesions, naming them "MWFL" and reporting, for the first time, a high prevalence associated with long-term use of acid-reducing drugs, specifically proton pump inhibitors (PPI) and H2-receptor antagonists (H2RA). ${ }^{3}$ However, these reports were conference presentations, and no studies on MWFL have been published to date. The present retrospective observational study aimed to clarify the endoscopic findings and clinicopathological features of MWFL.

\section{MATERIALS AND METHODS}

\section{Study design}

This was a retrospective, single-center, observational study. 


\section{Study period and subjects}

Subjects were consecutive patients who underwent upper gastrointestinal endoscopy as part of routine screening between April 2014 and March 2015 at Ihara Kasuga Clinic, affiliated with Fukuoka University Chikushi Hospital. Exclusion criteria were previous total gastrectomy and insufficient details in the medical notes regarding the history of acid-reducing drug use.

\section{Endoscopic procedures}

A single endoscopist (KY) with 15 years of experience with magnifying gastroscopy performed all endoscopic diagnostic procedures. The instruments used in the procedures were high-resolution magnifying upper gastrointestinal endoscopes (GIF-Q240Z or GIF-H290Z; Olympus, Tokyo, Japan) and an endoscopy system (EVIS LUCERA SPECTRUM; Olympus). In addition, a black soft hood (MAJ 1989; Olympus) was mounted at the tip of the scope in order to consistently fix the focal distance between the tip of the scope and the mucosal surface during magnifying observation at the maximal magnification rate. At 30 minutes prior to the endoscopic examination, patients drank $100 \mathrm{~mL}$ of a preparation solution that contained 10,000 units of pronase (Kaken Pharmaceutical, Tokyo, Japan), $1 \mathrm{~g}$ of sodium bicarbonate, and $10 \mathrm{~mL}$ of dimethylpolysiloxane $(20 \mathrm{mg} / \mathrm{mL}$; Horii Pharmaceutical Ind., Osaka, Japan). After inserting the endoscope, all of the gastric mucosa was inspected according to the systematic screening protocol for the stomach (SSS). ${ }^{4}$ When the SSS was completed, detected mucosal lesions were observed by conventional white-light imaging, non-magnifying narrow-band imaging (N-NBI), and magnifying narrow-band imaging (M-NBI) at the maximum magnification rate. All endoscopic images were recorded in an image filing system. A target biopsy was taken from the MWFL.

\section{Endoscopic image analysis}

The conventional white-light, N-NBI, and M-NBI images recorded in the endoscopic image filing system were reviewed and analyzed regarding the presence or absence and location of any MWFL and related endoscopic findings and the presence or absence of background mucosal atrophy.

\section{Histopathological analysis}

Endoscopic biopsy specimens were fixed in $20 \%$ formalin and embedded in paraffin. After hematoxylin and eosin staining, sections were reviewed and histopathological features were analyzed.

\section{Analysis of patient characteristics}

Medical record data were reviewed. Age, sex, presence or absence of background mucosal atrophy, presence or absence of Helicobacter pylori infection, use or non-use of acid-reducing drugs, and duration of any acid-reducing drug use were compared between patients with and without MWFL.

\section{Background mucosal atrophy analysis}

Kimura-Takemoto classification was applied to background mucosal atrophy observed on white-light images. ${ }^{5}$ Discoloration and a visible vascular pattern in the mucosa of the lesser curvature of the gastric angle were defined as atrophy.

\section{H. pylori infection analysis}

H. pylori infection was diagnosed based on wide areas of mucosal atrophy on endoscopic images as well as positive results on serum anti-H. pylori immunoglobulin G antibody test, urea breath test, or microscopy findings. For patients who had not undergone these tests, observation of wide areas of mucosal atrophy on endoscopic images alone was used to determine $H$. pylori infection. Previous $H$. pylori eradication therapy was also used as a marker of $H$. pylori infection.

\section{Statistical analysis}

Continuous variables, presented as mean \pm standard deviation, were compared using Student's $t$-test. Categorical variables were compared using Fisher's exact test. $P$-values less than 0.05 were considered significant. Statistical analyses were performed using the statistical software package SPSS 16.0 for Windows (SPSS Inc., Chicago, IL, USA).

\section{RESULTS}

After excluding 1 patient with previous total gastrectomy and 1 patient whose medical notes did not include a detailed history of oral drug use, 177 of the 179 consecutive patients who underwent screening were analyzed. MWFL were observed in 13 patients (7.3\%).

Endoscopic findings of the MWFL were as follows. On conventional white-light imaging, multiple whitish, flat, slightly elevated lesions of various sizes were observed in the gastric body and fundus (Fig. 1A). The boundaries between each lesion and the background mucosa were clearly demarcated. With N-NBI imaging, the background mucosa appeared dark brownish, increasing the contrast with the white lesions and making them easier to visualize (Fig. 1B). On M-NBI, clear demarcation lines were observed at the base of the flat elevated lesions. No superficial microvascular patterns were visible (Fig. 1C). In terms of microsurface patterns, the marginal crypt epithelium was uniformly long, narrow, and elliptical. The width of the marginal crypt epithelium was large, and it 
increased slightly and presented a faint dark brown color in the intervening part between crypts.

Target biopsy was performed in 4 of the 13 patients with MWFL. Histopathological analysis revealed hyperplastic changes of the foveolar epithelium in all biopsy specimens (Fig. 2A). Regarding the histopathological findings of MWFL, a hyperplastic change was defined as "moderate to marked" when the width of the crypt epithelium was $\geq 1.5$ times that in the background mucosa and as "mild" when the width of the crypt epithelium was $<1.5$ times that in the background mucosa.

These changes were mild in 1 patient and moderate to marked in 3 patients. In addition, parietal cell protrusions and oxyntic gland dilatations were observed in the fundic glands of 3 of the 4 patients (Fig. 2B). No intestinal metaplasia was observed.

The mean age of the 13 patients with MWFL was 74.5 years. Lesions occurred in the fundus only $(n=11)$ and from the fundus across the gastric body $(n=2)$. Background mucosal atrophy was present in 4 patients (30.8\%). Table 1 shows the mean ages, sex ratios, rates of background mucosal atrophy, presence or absence of $H$. pylori infection, and rates and mean durations of acid-reducing drug use in patients with and without MWFL. MWFL were observed in 3 men and 10 women and not observed in 80 men and 84 women, showing a tendency toward a higher prevalence in women $(p=0.089)$. H. pylori infection was present in $7.7 \%(1 / 13)$ of patients with MWFL and $42.7 \%$ (70/164) of patients without. Thus, sig-
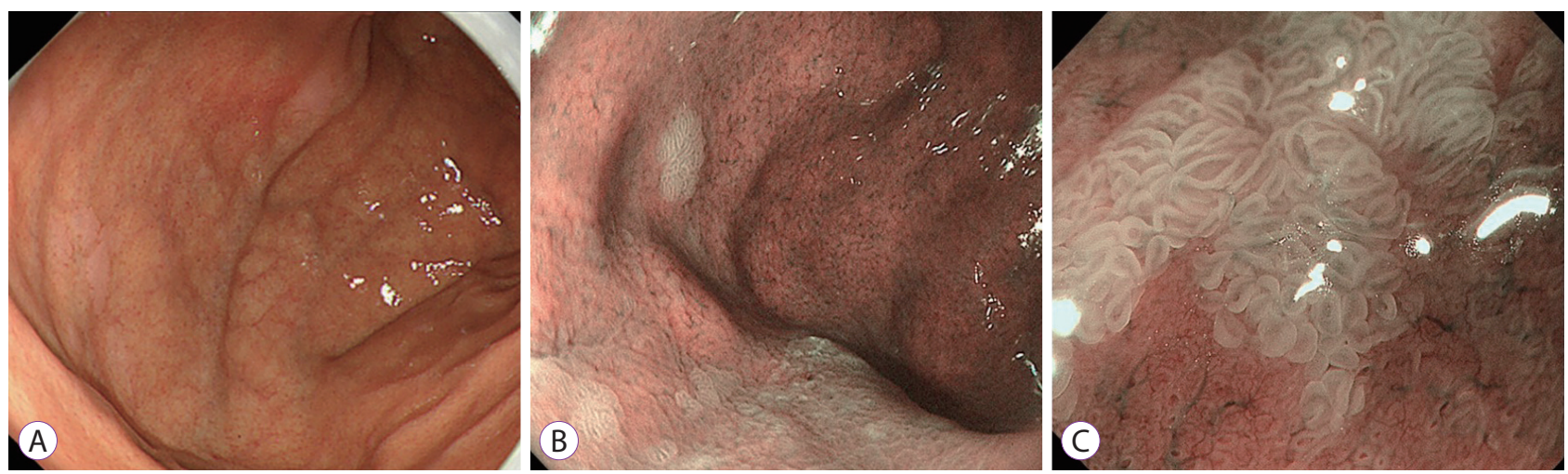

Fig. 1. Endoscopic findings of multiple whitish flat elevated lesions. (A) Conventional white-light imaging. Multiple whitish, flat, slightly elevated lesions of various sizes can be seen in the gastric fundus. (B) Non-magnifying narrow-band imaging. The background mucosa appears dark brownish, increasing the contrast with the white lesions and making them easier to visualize. (C) Magnifying narrow-band imaging. Clear demarcation lines can be observed. The superficial microvascular pattern is not clearly visualized. In terms of microsurface patterns, the marginal crypt epithelium is large, increasing slightly and presenting a faint dark brown color in the intervening part between crypts.
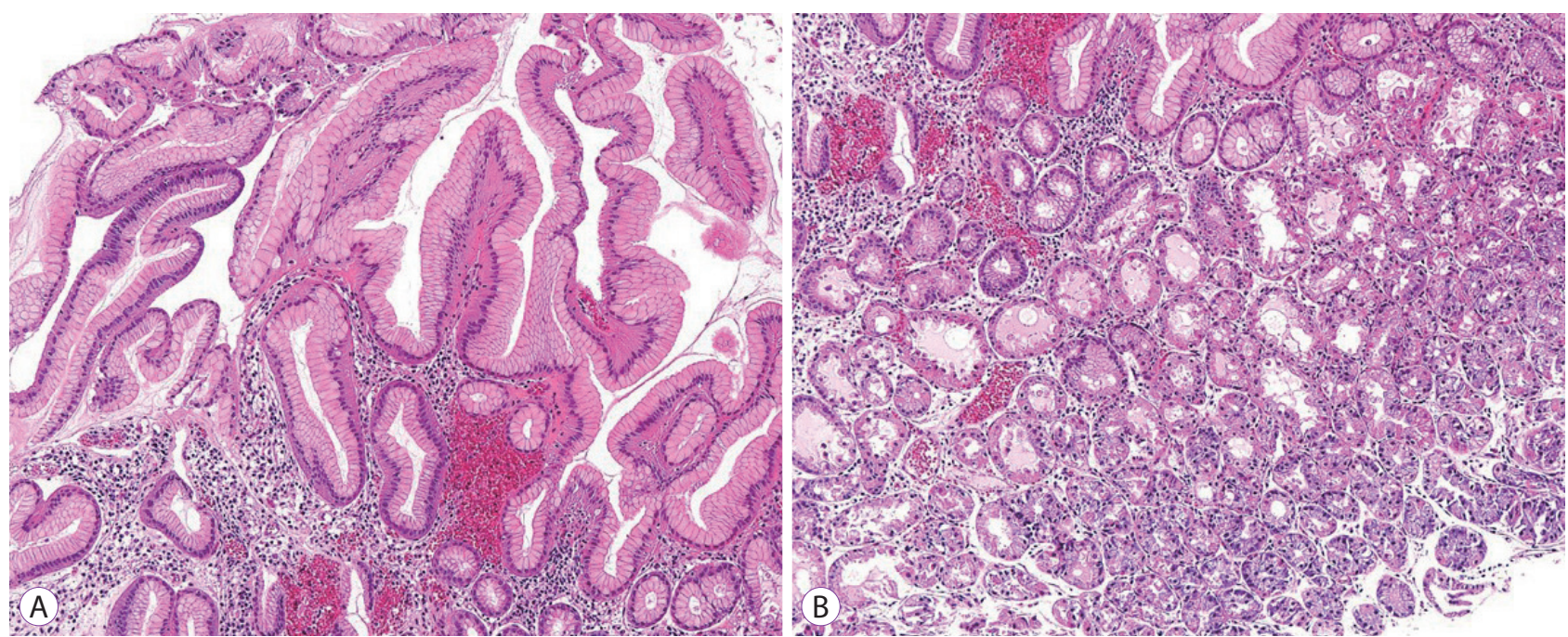

Fig. 2. Histopathological findings of biopsy specimen (hematoxylin and eosin staining). (A) Hyperplastic changes can be seen in the foveolar epithelium. (B) Parietal cell protrusions and oxyntic gland dilatations can be seen. 
nificantly more patients with MWFL did not have $H$. pylori infection compared to those without MWFL $(p=0.016)$. Of the 13 patients with MWFL, only 1 patient was infected with H. pylori, and the patient did not receive eradication therapy for H. pylori.

Furthermore, 100\% (13/13) of patients with MWFL used acid-reducing drugs, with a mean duration of use of 3.7 years. Among the types of acid-reducing drugs, oral PPI were used in 12 patients and oral H2RA were used in 1 patient. Of 92 patients with PPI use in this study, 12 (13\%) had MWFL; of 9 patients with H2RA use, 1 (11.1\%) had MWFL. No significant difference was noted in the frequency of MWFL according to the type of acid-reducing drug used (Table 2).

Conversely, the rate of acid-reducing drug use in patients without MWFL was 53.7\% (88/164), with a mean duration of 4.7 years. The rate of acid-reducing drug use was significantly higher in patients with compared to those without MWFL $(p<0.001)$. No significant differences were observed between patients with and without MWFL with regard to age, rates of background mucosal atrophy, and mean duration of acid-reducing drug use (Table 1).

\section{DISCUSSION}

\section{Key results}

The rate of acid-reducing drug use was significantly higher in patients with MWFL compared to those without (100\% [13/13] vs. $53.7 \%$ [88/164], $p<0.001)$.

\section{Discussion of key results}

Previous studies on PPI-related gastric lesions have reported fundic gland polyps, hyperplastic polyps, and hemorrhagic polyps formed like fundic gland polyps. ${ }^{6,7}$ Furthermore, diffuse changes associated with PPI use, specifically gastric cracked and cobblestone-like mucosa, have also been reported in the region of the gastric corpus glands. ${ }^{8}$ Although the high prevalence of MWFL in long-term PPI users has been reported in conference abstracts and Japanese language review articles, to our knowledge, this is the first original English language paper to examine the presence, endoscopic features, and histopathological findings of MWFL. ${ }^{9,10}$ The present findings indicated a correlation between MWFL and acid-reducing drug use.

The pathology of MWFL remains to be clarified. The elevated portion of these polyps is primarily formed from hyperplastic changes of the foveolar epithelium. Histologically, the biopsy specimens from the MWFL in the present study included fundic gland parietal cell protrusions and oxyntic gland dilatations. These findings differ completely from the previously reported PPI-related fundic gland polyps, hyperplastic polyps, and hemorrhagic polyps formed like fundic gland polyps. It is unclear whether the present histological changes were due to hypergastrinemia or represented the direct effects of PPI.

Endoscopic findings similar to the present gastric fundus MWFL have been previously reported as intestinal metaplasia; however, examination of the histopathological findings from the present MWFL biopsy specimens showed no findings of

Table 1. Comparison of Patient Characteristics and Clinical Presentation between Patients with and without Multiple White Flat Lesions

\begin{tabular}{|c|c|c|c|}
\hline & With MWFL & Without MWFL & $p$-value \\
\hline Age $(\mathrm{yr})( \pm \mathrm{SD})$ & $74.5( \pm 7.2)$ & $67.6( \pm 14.3)$ & $\mathrm{NS}^{\mathrm{a})}$ \\
\hline Sex (men:women) & $3: 10$ & $80: 84$ & $0.089^{b)}$ \\
\hline Rate of background mucosal atrophy & $30.8 \%(4 / 13)$ & $42.1 \%(69 / 164)$ & $\mathrm{NS}^{\mathrm{b})}$ \\
\hline Rate of Helicobacter pylori infection & $7.7 \%(1 / 13)$ & $42.7 \%(70 / 164)$ & $0.016^{\mathrm{b})}$ \\
\hline Rate of acid-reducing drug use & $100 \%(13 / 13)$ & $53.7 \%(88 / 164)$ & $<0.001^{\mathrm{b})}$ \\
\hline Mean (range) duration of acid-reducing drug use (yr) & $3.7(1-11)$ & $4.7(0.2-13)$ & $N S^{b)}$ \\
\hline
\end{tabular}

MWFL, multiple whitish flat elevated lesions; SD, standard deviation; NS, not significant.

${ }^{a)}$ Student- $t$ test; ${ }^{\text {b) }}$ Fisher's exact test.

Table 2. Numbers of Patients with MWFL among Those with PPI use and Those with H2RA Use

\begin{tabular}{|c|c|c|c|}
\hline & \multicolumn{2}{|c|}{ MWFL } & \multirow{2}{*}{$p$-value } \\
\hline & $(+)$ & $(-)$ & \\
\hline PPI & 12 & 80 & \multirow{2}{*}{$\mathrm{NS}^{\mathrm{a})}$} \\
\hline H2RA & 1 & 8 & \\
\hline
\end{tabular}

MWFL, multiple whitish flat elevated lesions; PPI, proton pump inhibitor; H2RA, H2-receptor antagonist; NS, not significant.

${ }^{\text {a) }}$ Fisher's exact test. 
intestinal metaplasia. Moreover, as previously stated, the elevated portions were formed only through hyperplasia of the foveolar epithelium. ${ }^{11-13}$ Therefore, histopathologically, MWFL are hyperplasia of the foveolar epithelium and not intestinal metaplasia.

\section{Limitations}

Limitations of the present study include the retrospective, single-center study design, the fact that biopsies were not performed from all identified polyps or background mucosa, and the lack of measurement of gastrin levels. Owing to the retrospective nature of our study, $\mathrm{H}$. pylori infection was determined in 99 (55.9\%) of 177 patients based on the findings of endoscopic imaging alone. Thus, $H$. pylori infection might not have been accurately determined.

\section{Future issues}

In order to overcome these limitations and clarify points that could not be verified in the present study, a prospective trial is already underway to investigate the relationship between MWFL and PPI use, serum gastrin levels, MWFL endoscopic findings, MWFL and background mucosa histopathological findings, and the characteristics of patients with MWFL (UMIN000030144). In this ongoing prospective trial, we will investigate $H$. pylori infection using results from 2 methods: the serum anti- $H$. pylori immunoglobulin G antibody test and microscopic examination. We will also attempt to clarify the association between $H$. pylori infection and the presence of MWFL using reliable data from reliable data from the present study. In the present study, the small number of patients precluded an investigation of the association between $H$. pylori infection and the presence of MWFL. In the future, we will investigate whether there are different features of MWFL between patients previously untreated and those treated with eradication therapy for $H$. pylori in a large prospective trial. Herein, we were unable to determine whether the discontinuation of acid-reducing drugs resulted in the regression of MWFL, which therefore also requires further clarification.
The present study indicated a relationship between the presence and endoscopic features of MWFL and a history of acid-reducing drug use.

\section{Conflicts of Interest}

The authors have no financial conflicts of interest.

\section{REFERENCES}

1. Turner K, Genta RM. The nonneoplastic stomach. In: Noffsinger A, ed. Fenoglio-Preiser's gastrointestinal pathology. 4th ed. Philadelphia (PA): Wolters Kluwer; 2017. p. 136-223.

2. Kawaguchi M, Arai E, Nozawa $\mathrm{H}$, et al. An investigation of white flat elevations in the gastric body. Gastroenterological Endoscopy 2007;49(Suppl 1):958.

3. Kamada T, Kawaguchi M, Maruyama Y, et al. New gastric lesion in the cardia induced by proton pump inhibitor treatment. Gastroenterology 2011:140(5 Suppl 1):S-719.

4. Yao K. The endoscopic diagnosis of early gastric cancer. Ann Gastroenterol 2013;26:11-22.

5. Kimura K, Takemoto T. An endoscopic recognition of the atrophic border and its significance in chronic gastritis. Endoscopy 1969;1:87-97.

6. Hongo M, Fujimoto K. Incidence and risk factor of fundic gland polyp and hyperplastic polyp in long-term proton pump inhibitor therapy: a prospective study in Japan. J Gastroenterol 2010;45:618-624.

7. Takeda T, Asaoka D, Tajima Y, et al. Hemorrhagic polyps formed like fundic gland polyps during long-term proton pump inhibitor administration. Clin J Gastroenterol 2017;10:478-484.

8. Miyamoto S, Kato M, Tsuda M, et al. Gastric mucosal cracked and cobblestone-like changes resulting from proton pump inhibitor use. Dig Endosc 2017;29:307-313.

9. Kamada T, Murao T, Osawa M, et al. A total of 379 multiple white and flat elevated lesions in the gastric fundus are induced by acid suppressive agents and long-term proton pump inhibitor treatment. Gastroenterology 2015;148(4 Suppl 1):S-319.

10. Haruma K, Shiotani A, Kamada T, et al. Problems of prolonged administration of PPI-occurrence of gastric polyps. Shōkaki naika 2013;56:190193.

11. Pimentel-Nunes P, Libânio D, Lage J, et al. A multicenter prospective study of the real-time use of narrow-band imaging in the diagnosis of premalignant gastric conditions and lesions. Endoscopy 2016;48:723730.

12. Pimentel-Nunes P, Dobru D, Libânio D, Dinis-Ribeiro M. White flat lesions in the gastric corpus may be intestinal metaplasia. Endoscopy 2017;49:617-618.

13. Uedo N, Yamaoka R, Yao K. Multiple white flat lesions in the gastric corpus are not intestinal metaplasia. Endoscopy 2017;49:615-616. 